\title{
Prevalence and predictors of smoking in a mining town in Kitwe, Zambia: A 2011 population-based survey
}

\author{
Cosmas Zyaambo ${ }^{1,2^{\star}}$, Olusegun Babaniyi ${ }^{3}$, Peter Songolo ${ }^{3}$, Adamson S. Muula $^{4}$, \\ Emmanuel Rudatsikira ${ }^{5}$, Seter Siziya ${ }^{6}$ \\ ${ }^{1}$ Department of Public Health, School of Medicine, University of Zambia, Lusaka, Zambia; \\ *Corresponding Author: czyaambo@yahoo.com \\ ${ }^{2}$ School of Public Health, The University of Alabama at Birmingham, Birmingham, USA \\ ${ }^{3}$ World Health Organization Country Office, Lusaka, Zambia \\ ${ }^{4}$ Department of Community Health, College of Medicine, University of Malawi, Blantyre, Malawi \\ ${ }^{5}$ School of Health Professions, Andrews University, Berrien Springs, Michigan, USA \\ ${ }^{6}$ Public Health Unit, Department of Clinical Sciences, School of Medicine, The Copperbelt University, Ndola, Zambia
}

Received 1 April 2013; revised 2 May 2013; accepted 1 June 2013

Copyright (C) 2013 Cosmas Zyaambo et al. This is an open access article distributed under the Creative Commons Attribution License, which permits unrestricted use, distribution, and reproduction in any medium, provided the original work is properly cited.

\section{ABSTRACT}

Smoking is one of the major preventable causes of death and non-communicable diseases which include hypertension, cardiovascular diseases and cancers. The aim of the study is to establish prevalence and predictors of smoking so that interventions specific to these communities can be executed to prevent smoking. A cross sectional study was conducted using a modified World Health Organizations Global Non Communicable Diseases (NCD) Surveillance Initiative NCD-STEPs 1 and 2. Multivariate logistic regression was used to examine the determinants of tobacco smoking. A total of 1627 individuals participated in the survey, of which 42.3\% were males. About half of the participants were of age 25 - 34 years (56.0\%), and $41.7 \%$ had attained secondary level of education. Overall, $8.7 \%$ of the participants $(18.1 \%$ among males and $1.8 \%$ among females) currently smoked any tobacco product. Female respondents were $71 \%$ $(A O R=0.29,95 \% \mathrm{Cl}[0.21,0.39])$ less likely to smoke cigarettes compared to male respondents. Compared to respondents who had no formal education, respondents who had attained primary level of education were $45 \%$ (AOR $=1.45$, $95 \% \mathrm{Cl}[1.02,2.08])$ more likely to smoke, and those who attained college or university level of education were $57 \%(A O R=0.43,95 \% \mathrm{Cl}[0.28$, $0.65]$ ) less likely to smoke. Respondents who did not consume alcohol were $50 \%$ (AOR $=0.50$, $95 \% \mathrm{Cl}[0.41,0.61])$ less likely to smoke compared to those who consumed alcohol. The study showed that sex, education, and alcohol consumption were independently associated with Smoking. These are the key determinants which should be considered when designing a health education and awareness campaign to the residents.

Keywords: Smoking; Health Education; Alcohol Consumption; Zambia

\section{INTRODUCTION}

Tobacco smoking is a major preventable cause of death and non-communicable disease globally [1-5]. According to the World Health Organization (WHO), it is estimated that 4 million deaths are attributable to tobacco smoking annually, and $70 \%$ of these deaths occur in developing countries [6]. Murray et al. [7] have projected that mortality related to tobacco use will increase from 3 million deaths in 1990 to 8.4 million deaths by 2020. In 2011, 6 million people died as a result of tobacco consumption worldwide. The number of tobacco-related deaths has tripled in the last decade, and if this trend continues, 1 billion people will die of tobacco use and exposure to environmental tobacco smoke in the 21st century at the rate of 1 person every 6 seconds [8]. Smoking has been perceived to be prevalent in developed countries, but this trend is changing, as tobacco smoking 
is also increasing in developing countries, particularly among men [9]. Zambia is no exception, as the prevalence of tobacco smoking among men has increased from $13.7 \%$ to $23.8 \%$ and among women from $0.5 \%$ to $0.7 \%$ over last decade [10]. HIV and AIDS are critical problems in Zambia which have resulted in great morbidity and mortality, however, as accessing to formal healthcare and particularly antiretroviral therapy services is increasing, life expectancy is improving, and the occurrence of chronic smoking-related diseases such as cancer and cardiovascular diseases is also expected to increase. Smoking surveillance and prevention should be top priorities in countries where tobacco smoking is increasing [11]. Many countries including Zambia are committed to implementing the WHO Framework Convention on Tobacco Control (FCTC), but Zambia is still grappling with the resources necessary to implement this control.

Most of the information on tobacco smoking in Zambia is obtained from adolescents who have completed the Global School-based Student Survey and the Global Youth Tobacco Survey [12,13], However, these data do not adequately address the issue of smoking in the older population, which is more susceptible to chronic diseases. A recent publication by Siziya et al. [14] presented such results from an older population, but only in the limited area of the Lusaka urban district; these findings cannot be generalized as the prevalence and determinants of tobacco smoking may vary between communities. It is therefore important to establish these determinants so that interventions specific to these communities can be executed to prevent smoking. The aim of this study is to determine the prevalence and predictors of smoking in a mining town in Kitwe, Zambia.

\section{MATERIALS AND METHODS}

\subsection{Setting and Design}

This was a cross sectional study which utilized a modified WHO Global Non-Communicable Disease surveillance initiative NCD-STEPs 1 and 2 in the city of Kitwe. Details of the survey methodology have already been published elsewhere [15]. However, we highlight some details of the methods that were used in the current survey in the following subsections.

\subsection{Sample Size and Sampling}

The sample size of the study was 1627 . It was determined using a prevalence of $50 \% \pm 5 \%$, a design effect of $2 \%$, a $95 \%$ response rate; and assuming an infinitely large population. A multi-stage sampling technique was used to draw the sample. The study area was divided into five constituencies: two constituencies comprising high cost residential areas, and three comprising low cost residential areas. One of the two high cost residentail areas and two of the three low cost residential areas were randomly selected at the first stage of sampling. A constituency was further divided into wards. At the second stage of sampling, one ward was randomly selected from each of the selected constituencies. A ward was further divided into Census Supervisory Areas (CSA). At the third stage of sampling, six CSAs from the low cost residential areas and four CSAs from the high cost residential areas were randomly selected. At the fourth stage of sampling, one Standard Enumeration Area (SEA) was selected from each selected CSA. Altogether, 10 SEAs were selected proportional to their population sizes. The line list of SEAs comprised the sampling frame and was obtained from the Central Statistics Office (2000 population census). From the selected SEAs, households were systematically sampled in order to widely cover the selected SEA. All individuals (male or female) aged 25 years or older in a household were eligible to participate in the study.

\subsection{Data Collection}

\section{Interviews}

An interview schedule was used to elicit responses from the interviewees. The questionnaire included the following sections: demographic information, tobacco smoking, alcohol consumption, sedentary behaviour, educational level and anthropometric measurements (including height and Weight). The questionnaire was administered in the respondents' homes by trained research assistants who included nurses and laboratory technologists.

\section{DATA MANAGEMENT AND ANALYSIS}

Data entry clerks were trained to enter the data using Epi Data software version 3.1. Data were double entered and validated. The data entry template had consistency and range checks embedded in it. The validated data was exported to SPSS version 11.5 for analysis. Missing data were declared as missing and were excluded from the analysis. The outcome variable was determined by using the question: Do you currently smoke any tobacco product, such as cigarettes, cigars or pipes? The response was either a Yes or No. Explanatory variables were age, sex, education, body mass index, sedentary behavior and alcohol consumption. The alcohol consumption variable was determined using the question: Have you consumed an alcoholic drink within the past 30 days? The responses were either Yes or No. Although the question on the number of standard alcoholic drinks consumed was asked, it was difficult to measure the quantity consumed by one participant because it is often the case in Zambia that more than one participant consume alcohol from the same container. Hence, we did not consider the quantity 
of alcohol consumed as another outcome variable Body Mass Index (BMI) was categorized as $<18.5$ (lean), 18.5 24.9 (normal), 25.0 - 29.9 (over weight) and 30+ (obese). Sedentary behavior was determined using the question: How much time do you usually spend sitting or reclining on a typical day? The response was recorded in hours and minutes; and was recoded into: $<1.5,1.5-3.4$ and 3.5+ hours. Proportions were compared using the Yates corrected Chi-square. Bivariate and multivariate logistic regression analyses were conducted. In multivariate analysis, a backward variable selection method was used. The cut off point for statistical significance was set at the $5 \%$ level.

\section{ETHICAL CONSIDERATIONS}

The study protocol was reviewed by the University of Zambia (UNZA) Biomedical Research Ethics Committee (BREC). Consent was obtained after the interviewer explained the purpose of the study to the eligible participant. Entry forms were viewed only by approved study personnel.

\section{RESULTS}

A total of 1627 individuals participated in the survey, of which $42.3 \%$ were males. About half of the participants were of age 25 - 34 years (56.0\%), and $41.7 \%$ had attained secondary level of education. Overall, $8.7 \%$ of the participants (18.1\% among males and 1.8\% among females) currently used any tobacco product. These results are presented in Table 1.

Table 1. Distributions of demographic characteristics and tobacco smoking status between sexes among Kitwe residents of Zambia.

\begin{tabular}{cccc}
\hline & Total & Male & Female \\
\hline Factor & $\mathrm{n}(\%)$ & $\mathrm{n}(\%)$ & $\mathrm{n}(\%)$ \\
\hline Age group (years) & & & \\
$25-34$ & $899(56.0)$ & $387(56.8)$ & $512(55.4)$ \\
$35-44$ & $346(21.6)$ & $127(18.6)$ & $219(23.7)$ \\
$45+$ & $360(22.4)$ & $167(24.5)$ & $193(20.9)$ \\
Education & & & \\
None & $78(4.9)$ & $22(3.3)$ & $56(6.0)$ \\
Primary & $499(31.1)$ & $160(23.7)$ & $339(36.5)$ \\
Secondary & $670(41.7)$ & $277(41.0)$ & $393(42.3)$ \\
College/university & $359(22.4)$ & $217(32.1)$ & $142(15.3)$ \\
Yurrently smoked cigarettes & & & \\
Yes & $140(8.7)$ & $123(18.1)$ & $17(1.8)$ \\
\hline & & $556(81.9)$ & $912(98.2)$ \\
\hline
\end{tabular}

When determining factors associated with tobacco smoking, sex, education, body mass index (BMI), and alcohol consumption were significantly associated with tobacco smoking in bivariate analyses (Table 2). On multivariate analysis, however, sex, education, and alcohol consumption were independently associated with tobacco smoking. Female respondents were 71\% (AOR $=0.29,95 \%$ CI $[0.21,0.39])$ less likely to use tobacco compared to male respondents. Compared to respondents who had no formal education, respondents who had attained primary level of education were 45\% (AOR = $1.45,95 \%$ CI $[1.02,2.08])$ more likely to smoke tobacco, and those who attained college or university level of education were $57 \%(\mathrm{AOR}=0.43,95 \% \mathrm{CI}[0.28,0.65])$ less likely to smoke tobacco. Respondents who did not consume alcohol were $50 \%(\mathrm{AOR}=0.50,95 \% \mathrm{CI}[0.41$, $0.61]$ ) less likely to use tobacco e compared to those who consumed alcohol.

Table 2. Factors associated with tobacco smoking in Kitwe.

\begin{tabular}{|c|c|c|}
\hline & Unadjusted & Adjusted \\
\hline Factor & OR (95\%CI) & AOR (95\%CI) \\
\hline \multicolumn{3}{|l|}{ Age (years) } \\
\hline $25-34$ & 1 & - \\
\hline $35-44$ & $0.99(0.79,1.25)$ & \\
\hline $45+$ & $0.77(0.56,1.05)$ & \\
\hline \multicolumn{3}{|l|}{ Sex } \\
\hline Male & 1 & 1 \\
\hline Female & $0.29(0.22,0.38)$ & $0.29(0.21,0.39)$ \\
\hline \multicolumn{3}{|c|}{ Completed level of education } \\
\hline None & 1 & 1 \\
\hline Primary & $1.13(0.84,1.52)$ & $1.45(1.02,2.07)$ \\
\hline Secondary & $0.89(0.66,1.18)$ & $0.81(0.57,1.14)$ \\
\hline College/university & $0.64(0.44,0.92)$ & $0.43(0.28,0.65)$ \\
\hline \multicolumn{3}{|l|}{ Body Mass Index (BMI) } \\
\hline$<18.5$ & 1 & - \\
\hline $18.5-24.9$ & $1.46(1.08,1.96)$ & \\
\hline $25.0-29.9$ & $0.77(0.52,1.13)$ & \\
\hline $30+$ & $0.36(0.21,0.63)$ & \\
\hline \multicolumn{3}{|c|}{ Consumed alcohol in past 30 days } \\
\hline Yes & 1 & 1 \\
\hline No & $0.36(0.30,0.44)$ & $0.50(0.41,0.61)$ \\
\hline \multicolumn{3}{|c|}{$\begin{array}{l}\text { Time usually spent sitting or } \\
\text { reclining on a typical day }\end{array}$} \\
\hline$<1.5$ & 1 & - \\
\hline $1.5-3.4$ & $1.12(0.88,1.42)$ & \\
\hline $3.5+$ & $0.84(0.65,1.08)$ & \\
\hline
\end{tabular}




\section{DISCUSSION}

This study uses the WHO STEP-wise approach to the surveillance of non-communicable disease datasets to analyze the prevalence and socio-demographic determinants of tobacco smoking in men and women aged above 25 years. For two cities of Zambia, Kitwe and Lusaka, we show that the prevalence of tobacco smoking in the former is higher [overall 8.7\% (18.1\% among males and $1.8 \%$ among females)] than what siziya et al. [14] found in the latter [overall 6.8\% (17.5\% among males and 1.5\% among females)] using the same methodology. A comparison of countries in which the same methodology was used shows that Zambia has lower smoking prevalence than the countries in the sub-Saharan region [16]. A recent publication has shown that the highest smoking rates are in developed counties, with developing counties having the lowest rates [17].

We show that, in Kitwe, sex, education, and alcohol consumption are the predictors of smoking. The higher prevalence of smoking in Kitwe could be due to the high rates of alcohol consumption. A study investigating alcohol consumption and its correlates in the same city showed a higher prevalence of alcohol consumption in Kitwe than in Lusaka [18]. Our results show that alcohol consumption is a predictor of smoking. A plausible explanation for this is that people who consume alcohol and smoke tobacco patronize the same bars, and hence the chances of a person who drinks starting to smoke tobacco are quiet high. We found that the prevalence of smoking was higher in men than in women. These results are consistent with those found in other countries [19,20]. In this regard, culture could play an important role in Zambia; it is not culturally acceptable for a woman to smoke or drink. Thus, with regard to smoking, women may not report their status accurately, and this may lead to inaccurate estimates of the prevalence. Women's socioeconomic status may also be a factor contributing to the low numbers of women who smoke; most women in Zambia do not have jobs and hence do not have sufficient disposable income to spend on tobacco products.

In our study, the respondent's level of education was found to be a key predictor of smoking after adjusting for age and sex. The more educated a respondent, the less likely he is or she is to smoking. However, in the developed countries, smoking was first indulged in by the elite and educated groups, and it then spread to the less educated [21]. This trend, however, is reversed for developing countries where the educated, understanding the health risks involved, tend to avoid smoking [22]. There is therefore a need to formulate smoking cessation strategies and increase the level of awareness on the dangers of smoking.

\section{STRENGTH AND LIMITATIONS}

The strength of this study lies in the fact that it's a cross section survey that involved a large population; it provides reliable and valid information on the subject. Apart from the prevalence of smoking, our study determined the risk factors associated with smoking. We do realize however that our study had some limitations that may make our results not generalizable to the entire population. The study did not have information on the number of household members of age of 25 years or older making it difficult for us to compute the response rates. We therefore could not compute weights that could be used in the analysis. Our results may be biased to the extent that the non-responses differed from those that participated in the study. We are however unable to suggest the direction of the bias. Vital information was not collected such as economic factors and, family history.

The study excluded adolescents less than 25 years of age; most of the studies compared with our study included this group. Our lower prevalence of smoking could be due to the absence of the adolescents in the analysis hence under estimating the prevalence in our current study.

\section{CONCLUSION}

Smoking is a risk factor for noncommunicable diseases which include hypertension, cardiovascular diseases and cancers. Despite the prevalence being low, there is a need to explore risk factors that may be associated with tobacco smoking. Health education and awareness campaign to the residents especially the miners at the working places are urgently needed. The regulation of smoking should be re-enforced and the taxes of tobacco products increased.

\section{ACKNOWLEDGEMENTS}

We are grateful to the participants, research assistants and the supervisors; without them this study would not have been done. We would like to extend our gratitude to the World Health Organization through the Zambian office and the Ministry of Health for funding this survey.

\section{REFERENCES}

[1] Ezzati, M., Henley, S.J., Lopez, A.D. and Thun, M.J. (2005) Role of smoking in global and regional cancer epidemiology: Current patterns and data needs. International Journal of Cancer, 116, 963-971. doi:10.1002/ijc.21100

[2] Tonstad, S. and Andrew Johnston, J. (2006) Cardiovascular risks associated with smoking: A review for clinicians. European Journal of Cardiovascular Prevention \& Rehabilitation, 13, 507-514. doi:10.1097/01.hjr.0000214609.06738.62 
[3] Mathers, C.D. and Loncar, D. (2006) Projections of global mortality and burden of disease from 2002 to 2030 . PLOS Medicine, 3, Article ID: e442. doi:10.1371/journal.pmed.0030442

[4] Ezzati, M., Lopez, A.D., Rodgers, A., Vander, S. and Murray, C.J. (2002) Major risk factors and global and regional burden of disease. Lancet, 360, 1347-1360. doi:10.1016/S0140-6736(02)11403-6

[5] Lopez A.D., Mathers, C.D., Ezzati, M., Jamison, D.T. and Murray, C.J. (2006) Global and regional burden of disease and risk factors, 2001: Systematic analysis of population health data. Lancet, 367, 1747-1757. doi:10.1016/S0140-6736(06)68770-9

[6] WHO (1999) Combating the tobacco epidemics: In the world health report. WHO, Geneva.

[7] Murray, C.J. and Lopez, A.D. (1997) Alternative projecttions of mortality and disability by cause 1990-2020: Global burden of disease study. Lancet, 349, 1498-1504. doi:10.1016/S0140-6736(96)07492-2

[8] Simon, S. (2012) Tobacco atlas: Tobacco kills 6 million in 1 year. American Cancer Society, USA. http://www.cancer.org/cancer/news/tobacco-atlas-tobacco -kills-6-million-in-1-year

[9] Mackay, J. (2002) The tobacco Atlas. WHO, Geneva.

[10] CSO (2003) Central Statistics Office, Lusaka, Zambia.

[11] WHO (2003) WHO framework convention on tobacco control. WHO, Geneva.

[12] Siziya, S., Rudatsikira, E., Muula, A.S. and Ntata, P.R. (2007) Predictors of cigarette smoking among adolescents in rural Zambia: Results from a cross sectional study from Chongwe district. Rural Remote Health, 7, 728.

[13] Muula, A.S. and Siziya, S. (2007) Prevalence and determinants of ever smoked cigarettes among school-going adolescents in Lusaka, Zambia. African Health Sciences, 7, 246-252.
[14] Siziya, S., Babaniyi, O., Songolo, P. and Nsakashalo-Senkwe, M. (2011) Prevalence and correlates for tobacco smoking among persons aged 25 years or older in lusaka urban district, Zambia. Journal of public Health and Epidemiology, 3, 43-48.

[15] Nsakashalo-Senkwe, M., Siziya, S., Goma, F.M., Songolo, P., Mukonka, V. and Babaniyi, O. (2011) Combined prevalence of impaired glucose level or diabetes and its correlates in Lusaka urban district, Zambia: A population based survey. International Archives of Medicine, 4, 2. doi:10.1186/1755-7682-4-2

[16] WHO (2013) Chronic disease and health promotion, STEPS country reports. http://www.who.int/chp/steps/reports/en/index.html

[17] WHO (2012) World health statistics report. World Health Oganisation, Geneva.

[18] Zyaambo, C., Babaniyi, O., Songolo, P., Muula, A., Rudatsikira, E. and Siziya, S. (2013) Alcohol consumption and its correlates among residents of mining town, Kitwe, Zambia. AMJ, 4, 260-265.

[19] Hosseinpoor, A.R., Parker, L.A., Tursan D’Espaignet, E. and Chatterji, S. (2011) Social determinants of smoking in low- and middle-income countries: Results from the World Health Survey. PLoS One, 6, Article ID: e20331. doi:10.1371/journal.pone.0020331

[20] Guindond, G.E. (2003) HNP discussion paper economics of tobacco control paper No. 6 past, current and future trends in tobacco use. The World Bank, Washington DC.

[21] Lopez, A.D. and Piha, T. (1994) A descriptive model of the cigarette epidemic in developed countries. Tobacco Control, 3, 242-247. doi:10.1136/tc.3.3.242

[22] Pampel, F. (2008) Tobacco use in sub-Sahara Africa: Estimates from the demographic health surveys. Social Science \& Medicine, 66, 1772-1783. doi:10.1016/j.socscimed.2007.12.003 